\title{
Functional MRI demonstrates pain perception in hand osteoarthritis has features of central pain processing
}

\author{
Nidhi Sofat ${ }^{1}$, Cori Smee ${ }^{1}$, Monika Hermansson ${ }^{1}$, Matthew Howard ${ }^{2}$, Emma H Baker ${ }^{1}$, Franklyn A \\ Howe $^{1,2}$, Thomas R Barrick ${ }^{1,2}$ \\ 1. Division of Biomedical Sciences, St George's, University of London, Cranmer Terrace, London, UK. 2. Institute of \\ Psychiatry, King's College London, de Crespigny Park, London, UK. 3. Centre for Stroke and Dementia Research, Division \\ of Clinical Sciences, St George's, University of London, Cranmer Terrace, London, UK.
}

Correspondence: Dr Nidhi Sofat. Address: Division of Biomedical Sciences, St George's, University of London, Cranmer Terrace, London, SW17 ORE. Email: nsofat@sgul.ac.uk.

Received: February 26, 2013

DOI : $10.5430 / j b g c . v 3 n 4 p 20$
Accepted: March 25, $2013 \quad$ Online Published: May 9, 2013

URL: http://dx.doi.org/10.5430/jbgc.v3n4p20

\section{Abstract}

Background: Hand osteoarthritis (HOA) is typified by pain and reduced function. We hypothesised that people with HOA have enhanced sensitivity and activation of peripheral nociceptors in the hand, thereby potentiating chronic pain. In our study we aimed to assess if central sensitisation mediates pain perception in osteoarthritis of the hand.

Methods: Participants with proximal and distal interphalangeal joint (PIP/DIP) HOA and non-OA controls were recruited. Clinical pain scores using the visual analogue scale (VAS) were recorded before and after performing a painful hand task. Central pain processing was evaluated with functional brain neuroimaging (fMRI) using a finger flexion-extension (FFE) task performed over 3 minutes. Data was analysed with FMRIB software (www.fmrib.ox.ac.uk/fsl). Group mean activation of functional MRI signal between hand osteoarthritis and control non-arthritic participants was compared.

Results: Our group of hand OA participants reported high pain levels compared with non-arthritic controls as demonstrated by the mean VAS in hand OA participants of $59.31 \pm 8.19 \mathrm{~mm}$ compared to $4.00 \pm 1.89 \mathrm{~mm}$ in controls $(p<$ 0.0001), despite all participants reporting analgesic use. Functional MRI analysis showed increased activation in the thalamus, cingulate, frontal and somatosensory cortex in the hand OA group but not in controls (thresholded at $p<0.05$ ). Regions of activation were mapped to Brodmann areas 3, 4, 6, 9, 13, 22, 24 and 44. Activated regions found in our study are recognised higher brain pain processing centres implicated in central sensitisation.

Conclusions: People with hand osteoarthritis demonstrated features of central sensitisation that was evident after a finger flexion-extension task using functional MRI. Functional MRI is a useful biomarker in detecting pain in hand osteoarthritis and could be used in future hand osteoarthritis pain studies to evaluate pain modulation strategies.

\section{Key words}

Hand osteoarthritis, Pain functional magnetic resonance imaging, Central sensitisation

\section{Introduction}

The central nervous system is an organ demonstrating plasticity in a system that has the capacity to change e.g. following peripheral tissue damage. Changes can occur at the molecular level and the neurotransmitter level ${ }^{[1]}$. Neurones may also 
alter their threshold of firing. Inflammation may lead to hypersensitivity of peripheral afferent neurones. As a result, neurones may be susceptible to increased firing, an effect also known as peripheral sensitisation ${ }^{[2,3]}$. In central sensitisation, there may be persistent activation of primary afferent neurones ${ }^{[4]}$. In chronic arthritis, a complex set of activation signals may then lead to the persistence of nociceptive pain. When activation signals reach a chronic phase, there is development of allodynia, which may be a pain sensation resulting from a non-painful stimulus and hyperalgesia, which is increased pain from a normally painful stimulus.

Osteoarthritis (OA) is the commonest form of arthritis worldwide, affecting increasing numbers of people in an ageing population ${ }^{[5]}$. In US adults, it is estimated that 27 million people have osteoarthritis ${ }^{[6]}$. The treatment of HOA includes analgesic drugs such as paracetamol, non-steroidal anti-inflammatory drugs (NSAIDs), opioid analgesics and rehabilitative hand physiotherapy ${ }^{[7,8]}$. Recent work has focused on aiming to understand mechanisms of pain in OA in the hope that deeper understanding of disease processes may lead to novel therapies ${ }^{[9]}$.

Recent work has focused on pain quantification in osteoarthritis. Several groups, including work in our unit, have reported the use of quantitative sensory testing (QST) ${ }^{[10-12]}$. Pain threshold testing using algometers has become more widely accepted for measuring pain perception objectively since it is reproducible over time and has been validated in large studies with knee OA ${ }^{[10]}$ or intra-oral pain ${ }^{[11]}$. We have found QST to be a useful objective measure of hand OA pain ${ }^{[12]}$. However, recent focus has interrogated imaging techniques to obtain a deeper understanding of pain processing in osteoarthritis. In recent years, several brain imaging techniques have been utilised to investigate arthritic pain. For example, fluoro-deoxy glucose positron emission tomography (FDG-PET) and functional magnetic resonance imaging (fMRI) have demonstrated that the perception of pain in OA is partly mediated by centrally-activated pathways in the brain ${ }^{[13-15]}$. Gwilym et al. ${ }^{[14]}$ reported increased activation of brain pain processing centres with fMRI in chronic hip OA, including the thalamus, anterior cingulate and insular cortex, upon quantitative sensory testing. Kulkarni et al. ${ }^{[13]}$ reported similar activation using FDG-PET in knee OA, suggesting activation of distinct brain regions in patients with chronic arthritic pain. Several authors have described the phenomenon of chronic pain centre activation during arthritis as central sensitisation, a process thought to derive from hypersensitivity to stimuli by long-term activation of peripheral receptors in arthritic joints.

We have now conducted a study in HOA to establish the characteristics of pain perception in participants with PIP (proximal interphalangeal joint) and DIP (distal interphalangeal joint) hand involvement. We hypothesised that pain perception in HOA is mediated by activation of local nociceptive stimuli in the hand causing sensitisation in chronic disease. We investigated our hypothesis by evaluating correlations between clinical measures of pain by VAS and comparing signal between hand OA participants and healthy non-OA controls by changes in fMRI signal observed during a painful hand task.

\section{Methods}

\subsection{Participants}

Full ethical approval for our study was obtained from The London-Surrey borders Research Ethics Committee, reference 09/H0718/60. Thirteen right-handed hand OA patients were recruited from rheumatology outpatient clinics at St George's Hospital, London as previously described ${ }^{[12]}$. All participants had hand pain due to primary OA of DIP (distal interphalangeal joints) and PIP (proximal interphalangeal) joints. Inclusion criteria were age range 40-75, female gender, being right-handed and fulfilling American College of Rheumatology (ACR) criteria for HOA. Exclusion criteria included another rheumatological diagnosis e.g. rheumatoid arthritis, recent surgery or metallic implants, male gender, diabetes mellitus, psychiatric disorders and other neurologic conditions. Thirteen right-handed controls were recruited through poster advertisements. All participants gave written informed consent and underwent the standard safety screening procedure for eligibility for MRI. 


\subsection{Clinical evaluation}

Participants in our study included people with an established diagnosis of hand osteoarthritis, as measured by ACR criteria, and non-arthritic controls who had no evidence clinically of osteoarthritis. The results of pain scores measured by visual analogue score (VAS) in relation to radiographic change of hands by Kellgren-Lawrence scoring, comparison of the hospital anxiety and depression score (HADS) and the health assessment questionnaire (HAQ) to assess hand function have previously been reported ${ }^{[12]}$. All the participants described in our published clinical study ${ }^{[12]}$ also underwent functional MRI scanning, the methods of which are described below.

In order to ensure validation of the study in view of the small sample size, a power analysis was conducted based on the mean VAS scores for pain obtained in each group. Power analysis was performed using an online power calculator http://www.dssresearch.com. Results showed based on VAS for the hand OA group $59.31 \pm 8.19 \mathrm{~mm}$ with $4.00 \pm 1.89 \mathrm{~mm}$ in controls, a sample size of $n=13$ in each group achieves a power of $100 \%$.

\subsection{Magnetic resonance image acquisition}

MRI was performed on a 1.5T GE MRI scanner (General Electric Systems, Milwaukee, USA) with an 8-channel head coil used for signal reception. An echo-planar imaging sequence was used to acquire blood-oxygenation level-dependent (BOLD) images with echo time (TE) $40 \mathrm{~ms}$, repetition time (TR) $3000 \mathrm{~ms}$ and a $90^{\circ}$ flip angle. Whole head coverage was achieved with $5 \mathrm{~mm}$ thick contiguous slices, a $64 \times 64$ acquisition matrix over a $210 \mathrm{~mm}$ field of view (nominal $3.3 \mathrm{~mm}$ in-plane resolution) and 1 signal average. 60 imaging volumes were collected over 3 minutes for the functional MRI paradigm. Each participant completed the paradigm twice during a single scanning session.

\section{Functional Magnetic Resonance I maging ( fMRI) Paradigm}

The fMRI task paradigm was a 3 minute block design where participants alternated periods of rest with a finger flexionextension (FFE) task with a 30 s period (see Figure 1a). The FFE task consisted of the participant repeatedly closing and opening their right hand over the 30s task period. The participant's arm was at rest by their side throughout all task periods. HOA participants reported that the task effectively mimics movements that induce pain. Control participants underwent the same fMRI protocol. Although 15 participants were initially recruited into each group, two HOA and two control participants were excluded from the final fMRI analysis because they performed the fMRI task incorrectly. This left a total number of 26 participants in the final analysis.

\section{Functional Magnetic Resonance I mage Analysis}

fMRI pre-processing and statistical analysis was performed using the Functional Software Library (FSL, http://www. fmrib.ox.ac.uk/fsl, Centre for Functional MRI of the Brain (FMRIB), Oxford University, UK). The following preprocessing steps were applied to each participant's fMRI time series: removal of non-brain structures using Brain Extraction Tool (BET) ${ }^{[16]}$, realignment of the fMRI time series using motion correction ${ }^{[17]}$ and brain registration.

Brain registration included normalisation of the fMRI time series to a standard brain image. This was performed using a 2-stage process to improve image registration to standard space. Firstly, each fMRI time series was coregistered to the Montreal Neurological Institute (MNI) 152 brain image (MNI152) using the FMRIB’s Linear Image Registration Tool (FLIRT) command which includes a 12 parameter affine transformation using the mutual information cost function. An average T2*-weighted image was then computed across all fMRI time series and all participants, providing a studyspecific atlas image in MNI space. Secondly, each fMRI time series was coregistered to the study specific atlas using FLIRT, as described in the first stage. This 2-stage process improved fMRI time-series normalisation by ensuring that registration was performed to a study-specific atlas image with similar field of view and image contrast. The regions of activation by BOLD signal were identified with Brodmann areas, which define the cerebral cortex into distinct structural regions. 
(a)
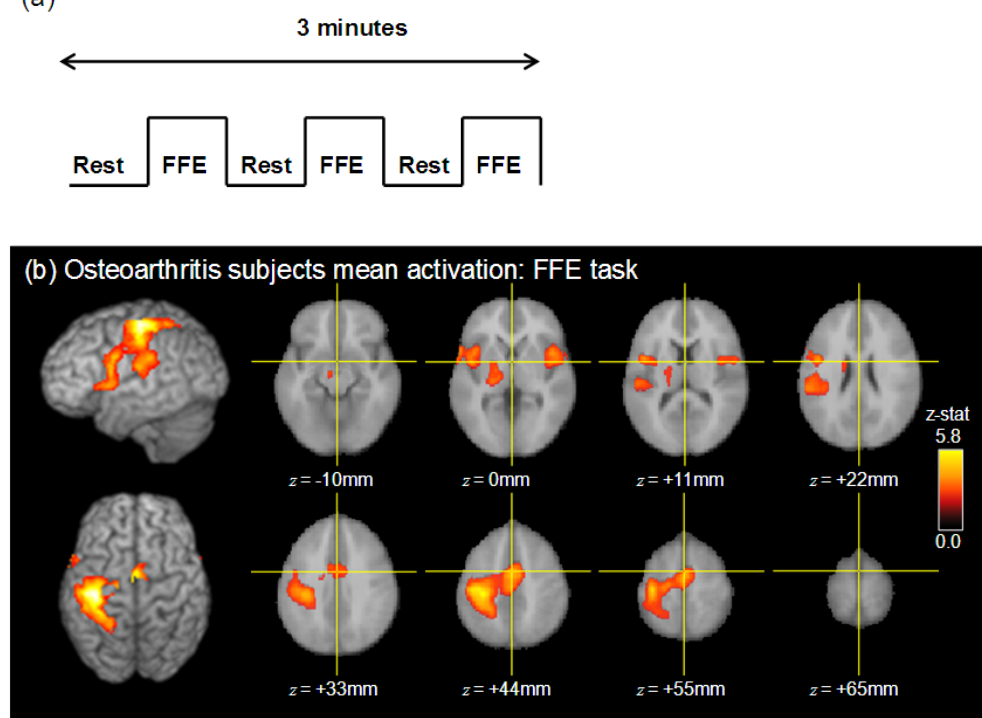

(c) Control subjects mean activation: FFE task

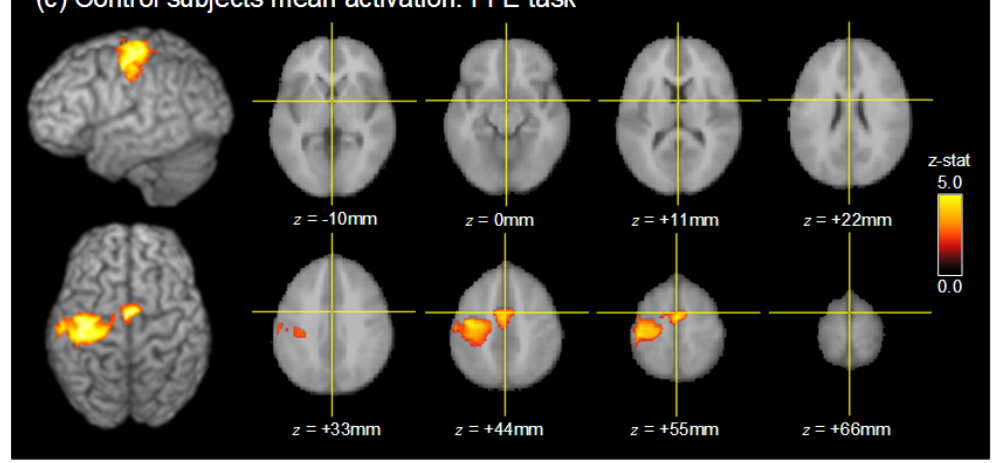

Figure 1. (a) Functional MRI acquisition paradigm with finger flexion-extension (FFE) task; (b) Mean activation in the HOA group measured by fMRI; (c) Mean activation in the control group measured by fMRI. All images are shown in neurological orientation with crosshairs, significance threshold 2.3 ( $p<0.05$ ), with areas of statistically significant increased activation, analysed using FMRIB software. Images are illustrated using MRIcro.

Statistical analysis was performed using the FMRIB Expert Analysis Tool (FEAT). First level analysis was performed with the following parameters: spatial smoothing using a Gaussian kernel of Full Width at Half Maximum (FWHM) of 8 $\mathrm{mm}$, each voxel time course was demeaned, and non-linear high-pass temporal filtering was applied at 60s. The initial two volumes of each fMRI time series were not statistically analysed due to T2*-weighted images not having reached full saturation prior to $6 \mathrm{~s}$ of scanning. Motion outliers were computed for each fMRI time series and their locations in the time series were entered as a confounding experimental variable. Intermediate level analysis was performed using a fixed effects analysis on both 3 minute trials per participant to obtain an average activation map for each participant. This was followed by a higher-level group analysis to obtain group mean activations for the control and HOA participants using a mixed effects FLAME 1 statistical analysis. A $t$-test was also performed and statistical inference was provided using a z-statistic threshold of 2.3 followed by multiple comparisons correction the cluster level using Family-Wise Error (FWE) at $p<0.05$.

\section{Results}

Central pain processing detected by fMRI demonstrates central pain sensitisation in hand OA. The visual analogue score (VAS) was used to detect participant's pain scores on a 0-100 mm scale. HOA participants reported high levels of pain, 
with a mean of $59.31+/-8.19$, compared with $4.00+/-1.89(p<0.0001)$ in controls. The HOA participants also had evidence of peripheral sensitisation measured by pressure algometry as previously reported ${ }^{[12]}$. In this study of the same cohort of participants we determined whether the enhanced pain perception reported by HOA participants was attributable to central (brain) pain sensitisation. In order to investigate central sensitisation, we designed a finger flexion extension (FFE) task to simulate naturally-evoked pain during fMRI (see Figure 1a). The FFE task had 30 sec intervals of rest followed by finger flexion and extension on a cyclical basis in order to evaluate fMRI BOLD activation signal.

During FFE, patients with HOA reported a mean pain VAS score of 55.92, which increased to 74.31 after the FFE task. In both HOA and control participants, there was a significant increase in BOLD signal on fMRI in primary motor and premotor cortices as would be expected with a motor task (Brodman Area (BA) 3/4/6) (see Figure 1b/c, Table 1). Both groups also demonstrated activation of the somatosensory cortex (BA 3/6), although the total area of the somatosensory cortex activated in HOA participants was greater than in controls, suggesting greater recruitment of higher brain sensory processing centres in HOA $(p<0.05)$. In HOA but not control participants, FFE induced a significant increase in BOLD signal on fMRI on measurement of group activation in regions that included the thalamus (which does not have a designated Brodman area), insula (BA 13) and cingulate cortex (BA 24) (see Figure 1b, 1c, Table 1a). These findings demonstrate that the FFE task simulating pain in hand OA participants activates additional brain regions recognised to mediate brain pain processing in addition to those activated by the same task in healthy controls.

Table 1a. Location of significant clusters and local maxima for functional activation maps obtained from the FFE task in hand OA group (see Figure 1b).

\begin{tabular}{llllll}
\hline $\begin{array}{l}\text { Cluster description } \\
\text { (size in voxels and volume) }\end{array}$ & p-value & Brodmann area & \multicolumn{4}{l}{ Talairach coordinate maxima (mm) } \\
\hline 1) $\left(9814,78.51 \mathrm{~cm}^{3}\right.$ ) & & 24 & 0 & -1 & 47 \\
Cingulate gyrus & 9 & -44 & 0 & 24 \\
Left hemisphere & 3 & -44 & -21 & 48 \\
Postcentral gyrus / Somatosensory cortex & $<0.001$ & 3 & -38 & -21 & 50 \\
Inferior frontal gyrus & & 3 & -32 & -23 & 44 \\
Right hemisphere & 6 & 2 & 0 & 54 \\
Medial frontal gyrus / Premotor cortex & & 22 & 58 & 5 & 54 \\
2) (998, 7.98 cm cm $_{\text {Right hemisphere }}$ & 22 & 56 & 6 & 0 \\
Superior temporal gyrus & 44 & 49 & 1 & 7 \\
Precentral gyrus & 0.0359 & 9 & 47 & 0 & 20 \\
Inferior frontal gyrus & & 13 & 43 & 7 & 4 \\
Insula & 13 & 41 & -4 & 9 \\
\hline
\end{tabular}

Table 1b. Location of significant clusters and local maxima for functional activation maps obtained from the FFE task in control group (see Figure 1c).

\begin{tabular}{|c|c|c|c|c|c|}
\hline \multirow{2}{*}{$\begin{array}{l}\text { Cluster description } \\
\text { (size in voxels and volume) } \\
\left(3940,31.52 \mathrm{~cm}^{3}\right)\end{array}$} & \multirow[t]{2}{*}{$p$-value } & \multirow{2}{*}{$\begin{array}{l}\text { Brodmann area } \\
3\end{array}$} & \multicolumn{3}{|c|}{ Talairach coordinate maxima (mm) } \\
\hline & & & -40 & -23 & 55 \\
\hline Left hemisphere & \multirow{5}{*}{0.003} & 4 & -40 & -17 & 55 \\
\hline Postcentral gyrus / Somatosensory cortex & & 6 & -39 & -12 & 43 \\
\hline Precentral gyrus / Primary motor cortex & & 4 & -39 & -15 & 49 \\
\hline Middle frontal gyrus / Premotor cortex & & 6 & -39 & -13 & 49 \\
\hline Medial frontal gyrus / Premotor cortex & & 6 & -1 & -5 & 51 \\
\hline
\end{tabular}




\section{Discussion}

Hand OA is a chronic disease in which mechanisms of pain are not fully understood. During the painful FFE task in hand OA participants, we detected fMRI activation signal in the thalamus, cingulate and insular cortex. Regions activated in both the HOA and control groups included the somatosensory and premotor cortex which would be expected in hand function and planning of movements. Previous investigators have also detected activation in the thalamus, cingulate and insular cortex in painful pressure paradigms for participants with OA knee ${ }^{[13]}$ and sensory testing for neuropathic pain in hip $\mathrm{OA}^{[14]}$. It is therefore significant that our study, the first of its kind in HOA, also showed significant activation in the thalamus, cingulate, and insular cerebral cortex but not controls. Of interest, the cingulate cortex is involved in developing emotion formation, learning and memory, suggesting that HOA participants are adapting their responses to sensory cues in their hand and developing unique pain activation systems compared with controls. Others have suggested that the cingulate cortex is important in mediating affective processing of pain ${ }^{[18]}$. The thalamus, which we found to be activated in our study, is a well recognised centre of central pain processing and is important in developing adaptive brain networks during chronic pain ${ }^{[19,20]}$. Our findings and those of others suggest that there are common central processing mechanisms in arthritic pain that are activated in several modalities of pain testing. Our data therefore suggest that central sensitisation observed in our HOA cohort reflect subjects' relatively high VAS scores for pain and lower pain thresholds measured by algometers. Our data also show that HOA participants may have a significant emotional component to their pain, since we observed BOLD signal activation in regions of the frontal cortex, also known as the dorsolateral prefrontal cortex (DLPFC) which processes emotion ${ }^{[20]}$. Parks et al. ${ }^{[21]}$ recently suggested differences between evoked pain (induced by a physical pressure) and spontaneous pain (without an external stimulus) in knee OA subjects ${ }^{[21]}$, suggesting that evoked pain has similar mechanisms in patients with knee OA and normal participants but that spontaneous pain is mediated by central sensitisation. The same group suggested that spontaneous pain is more likely to be modulated by intervention than evoked pain. In our model, one might envisage how spontaneous pain is akin to the VAS reporting by subjects and evoked pain is more similar to the algometer-induced nociceptve pain.

Limitations of our study include that we analysed a relatively small number of right-handed participants $(\mathrm{n}=26)$ presenting to secondary care, and these patients by their very nature are likely to have had more severe OA. The majority of our participants were also taking analgesics and could therefore have altered fMRI changes as a result.

\section{Conclusion}

People with hand osteoarthritis demonstrated features of central sensitisation that was evident after a finger flexionextension task using functional MRI. Functional MRI is a useful biomarker in detecting pain in hand osteoarthritis and could be used in future hand osteoarthritis pain studies to evaluate pain modulation strategies.

\section{Source(s) of support}

We thank the Rosetrees' Trust, St George’s Charitable Trust, The Wellcome Trust VIP award to Monika Hermansson and the London Comprehensive Research Network for financial support in conducting this study.

\section{References}

[1] Grassi F, Mileo AM, Monaco L, Punturieri A, Santoni A, Eusebi F. TNF alpha increases the frequency of spontaneous miniature synaptic currents in cultured rat hippocampal neurons. Brain Res. 1994; 659: 226-30. http://dx.doi.org/10.1016/0006-8993(94)90883-4

[2] Kidd BL, Urban LA. Mechanisms of inflammatory pain. Br J Anaes. 2001; 87: 3-11. PMid:11460811 http://dx.doi.org/10.1093/bja/87.1.3

[3] Eide PK. Wind-up and the NMDA receptor complex form a clinical perspective. Eur J Pain. 2000; 4: 5-15. PMid:10833550 http://dx.doi.org/10.1053/eujp.1999.0154 
[4] Dickenson AH, Sullivan AF. Evidence for a role of the NMDA receptor in the frequency dependent potentiation of deep rat dorsal horn nociceptive neurones following C-fibre stimulation. Neuropharmacology. 1987; 26: 1235-8. http://dx.doi.org/10.1016/0028-3908(87)90275-9

[5] Hunter DJ, Felson DT. Osteoarthritis. BMJ 2006; 18: 332: 640-2

[6] Lawrence RC, Felson DT, Helmick CG, et al. Estimates of the prevalence of osteoarthritis and other rheumatic conditions in the United States. Part II. Arthritis Rheum. 2008; 58 (1): 26-35. PMid:18163497 http://dx.doi.org/10.1002/art.23176

[7] Conaghan PG, Dickson J, Grant RL et al.Guideline Development Group. Care and management of osteoarthritis in adults: summary of NICE guidance. BMJ. 2008; 336(7642): 502-3. PMid:18310005 http://dx.doi.org/10.1136/bmj.39490.608009.AD

[8] Zhang W, Doherty M, Leeb BF, Alekseeva L, Arden NK, Bijlsma JW, Dinçer F, et al. EULAR evidence based recommendations for the management of hand osteoarthritis: report of a Task Force of the EULAR Standing Committee for International Clinical Studies Including Therapeutics (ESCISIT). Ann Rheum Dis. 2007; 66(3): 377-88. PMid:17046965 http://dx.doi.org/10.1136/ard.2006.062091

[9] Sofat N, Ejindu V, Kiely P. What makes OA painful? The evidence for peripheral and central pain processing. Rheumatology (Oxford). 2011; 50(12): 2157-65. PMid:21954151 http://dx.doi.org/10.1093/rheumatology/ker283

[10] Wylde V, Palmer S, Learmonth ID, Dieppe P. Test-retest reliability of Quantitative Sensory Testing in knee osteoarthritis and healthy participants. Osteoarthritis Cartilage. 2011; 19(6): 655-8. PMid:21329759 http://dx.doi.org/10.1016/j.joca.2011.02.009

[11] Pigg M, Baad-Hansen L, Svensson P, Drangsholt M, List T. Reliability of intraoral quantitative sensory testing (QST). Pain. 2010; 148: 220-6. PMid:20022428 http://dx.doi.org/10.1016/j.pain.2009.10.024

[12] Wajed, J, Ejindu V, Heron C, Hermansson, Kiely P, Sofat N. Quantitative sensory testing in painful hand osteoarthritis demonstrates features of peripheral sensitisation. International Journal of Rheumatology. 2012: 703138

[13] Kulkarni B, Bentley DE, Elliott R, Julyan PJ, Boger E, Watson A, Boyle Y, El-Deredy W, Jones AK. Arthritic pain is processed in brain areas concerned with emotions and fear. Arthritis Rheum. 2007; 56: 1345-54. PMid:17393440 http://dx.doi.org/10.1002/art.22460

[14] Gwilym S, Keltner JR, Warnaby CE, Carr AJ, Chizh B, Chessell I, Tracey I. Psychosocial and Functional Imaging Evidence Supporting the Presence of Central Sensitization in a Cohort of Osteoarthritis Patients. Arthritis Rheum. 2009; 61(9): 1226-1234. PMid:19714588 http://dx.doi.org/10.1002/art.24837

[15] Baliki MN, Geha PY, Jabakhanji R, Harden N, Schnitzer TJ, Apkarian AV. A preliminary fMRI study of analgesic treatment in chronic back pain and knee osteoarthritis. Mol Pain. 2008; 4: 47. PMid:18950528 http://dx.doi.org/10.1186/1744-8069-4-47

[16] Smith SM. Overview of fMRI analysis. Br J Radiol. 2004; 77: S167-75. PMid:15677358 http://dx.doi.org/10.1259/bjr/33553595

[17] Jenkinson M, Smith S. A global optimisation method for robust affine registration of brain images. Med Image Anal. 2001; 5: 143-156. http://dx.doi.org/10.1016/S1361-8415(01)00036-6

[18] Hsieh JC, Belfrage M, Stone-Elander S, Hansson P, Ingvar M. Central representation of chronic ongoing neuropathic pain studied by positron emission tomography. Pain. 1995; 63: 225-36. http://dx.doi.org/10.1016/0304-3959(95)00048-W

[19] Atlas LY, Bolger N, Lindquist MA, Wager TD. Brain mediators of predictive cue effects on perceived pain. J Neurosci. 2010; 30(9): 12964-77. PMid:20881115 http://dx.doi.org/10.1523/JNEUROSCI.0057-10.2010

[20] Kupfermann I. Localisation of higher cognitive and affective functions: the association cortices. In: Kander ER, Schwartz JH, Jessell TM, editors. Principles of neural science. Amsterdam: Elsevier; 1991. pp 823-38

[21] Parks EL, Geha PY, Baliki MN. Brain activity for chronic knee osteoarthritis: dissociating evoked pain from spontaneous pain. Eur J Pain. 2011; 15(8): 843.e1-14 